\title{
Natural and Human Resources that Support the Development of Curative Tourism in Albanian Territories
}

\author{
Dr. Albana Zejnel Hoxha \\ University "Aleksandër Xhuvani", Department of History-Geography, Elbasan, Albania \\ Email: albanazej@yahoo.it
}

\section{Doi:10.5901/ajis.2015.v4n3s1p161}

\section{Abstract}

\begin{abstract}
Tourism is one of the main directions of economic development of Albania, Kosovo and other albanian regions. Tourism assets of albanian regions are suitable for the development of diverse tourist activities, among which curative tourism. This paper aims to identify the location, natural features and curative value of thermal and thermal-mineral springs of albanian territories. Human resources that support the development of this kind of tourism are also taken into consideration. The study is based on available publications, fieldwork results, statistical sources and maps have been designed, generating conclusions and suggestions addressed to the heads of local governments, communities and entities that intend to invest in the development of sustainable curative tourism, according to the most successful contemporary models. At a time when tourism is considered as one of the most perspective branches in our region, becomes important the role that curative tourism plays in areas with curative tourism potential. According to the importance that it has, it is necessary to intensify efforts to promote: the enhancement of natural and human resources; policies and strategies for the development of curative tourism; development of curative infrastructure based on European standards; curative values left in limbo; preparation of guides etc.
\end{abstract}

Keywords: curative tourism, tourism potential, natural resources, human resources, promotion etc.

\section{Introduzione}

II turismo curativo consiste nello spostamento di una categoria di persone dalla propria abitazione verso altri luoghi al fine del miglioramento delle anomalie fisiologiche e della cura di una malattia.

Alcuni autori definiscono le destinazioni turistiche ed i vari tipi del turismo curativo in base alla motivazione e al luogo:

- Turismo curativo tradizionale che consiste nello sfruttamento delle acque termo- minerali. Gli inizi di questo tipo di turismo risalgono all'antichitá. I luoghi dove viene applicato si chiamano terme (llixha).

- Turismo curativo montano legato all'aria pura e il clima salutare. Questo tipo di turismo si applicca principalmente da persone affette di malattie dell' apparato respiratorio, dagli anziani e da quelli che vogliono avere una salute sana.

- Turismo curativo del benessere, il quale alterna il turismo curativo tradizionale con degli elementi di ricreazione e di divertimento. Si applica specialmente da persone con un reditto più alto della media.

- Turismo balneare (talassoterapia), consiste nello sfruttamento dei vantagggi che offre il mare, le spiaggie e il clima costiero per il riposo e il miglioramento della salute sotto l'effetto del sole, della sabbia e dell'acqua.

- Turismo terapeutico che ha avuto inizio alla fine del ventesimo secolo, si applica principalmente da persone con redditi alti, che frequentano i centri medico - turistici per mantenere il corpo in buona forma, addirittura per assicurare I'"eterna giovinezza ". I trattamenti includono i massaggi e gli interventi chirurgici ${ }^{1}$.

Il turismo curativo oggi si identifica con il movimento delle persone verso i centri curativi non solo per il trattamento curativo delle patologie, ma anche per garantire il benessere del corpo e della mente (Becheri 2007). Recentemente in questo campo sono inclusi termini quali SPA (termale) e wellnes ( benessere). Lo sviluppo e la fioritura di tutte le forme del turismo curativo vengono sostenuti dalle risorse naturali e umane che offrono le aree terapeutiche.

\footnotetext{
${ }^{1} \mathrm{http}: / / w w w . d e c . u n i c h . i t$
} 


\section{Le Risorse Naturali, la Base per lo Sviluppo del Turismo Curativo}

L'offerta geografica-naturale rappresenta la base della potenzialitá turistica. Essa trova sostegno nelle risorse naturali che influenzano direttamente lo sviluppo del turismo (Dh. Doka, B. Draçi 2009). I fattori naturali sostenitori del turismo curativo termale sono:

- La formazione geologica dei territori albanesi. I territori albanesi con una posizione geologica favorevole sono ricchi di sorgenti termo-minerali. Queste sorgenti sono strettamente legate alla costruzione geologica, alla storia geologica delle tracce di un ex vulcanismo. Questa costruzione fornisce la ricca composizione minerale-chimica delle acque termali minerali aumentando le loro qualità curative.(L'aerea geotermale di Kruja, di Peshkopi e di Ardenica) (A. Frashëri,V. Cermak et al, 2004)

- La ricchezza idrica. Lo sviluppo del turismo curativo è direttamente legata ad essa. Sono esattamente le numerose sorgenti termali e minerali che hanno permesso sin dal passato il movimento della popolazione verso queste sorgenti per curare le malattie, per garantire il benessere dell' organismo.

- II clima e l'aria pura. II movimento dei turisti, la durata del loro soggiorno sono strettamente legati al clima delle regioni in cui sono concentrate le sorgenti termali. Parte dei servizi termali e le strutture ospitanti sono circondati da superficie boscose, offrendo ai visitatori l'aria pulita e salubre.

II clima dell'Albania è tipicamente mediterraneo. II movimento turistico dipende da queste condizioni climatiche. Ciò si riflette nel flusso dei visitatori durante le stagioni dell'anno. I centri curativi si frequentano soprattutto durante la primavera e l'autunno. Durante queste due stagioni l' afflusso dei visitatori varia in media da 2500 a 6000.

Alcuni fattori di rilevante importanza per lo sviluppo del turismo curativo sono anche il rilievo montuoso, i mari ed i laghi. Il clima puro e salubre delle zone montuose favorisce il movimento turistico verso esse. Prendere il sole e curarsi di sabbia spesso appaiono più attraenti di altre offerte naturali.

\subsection{Le risorse termali e minerali in Albania e negli altri territori albanesi}

L'Albania, II Kosovo e gli atlri territori albanesi offrono delle considerevoli potenzialitá naturali per lo sviluppo del turismo curativo. Le principali risorse delle acque termali si trovanano a

- Elbasan, le sorgenti delle Llixhave Qendrore (le Terme), Hidraj, Kozan (il pozzo geotermale);

- Peshkopi, le sorgenti all'est della cittá;

- Përmet, le sorgenti termali di Bënjë;

- Fushë Krujë, le sorgenti termali al villaggio Bilaj;

- Leskovik, le sorgenti di Sarandoporo,Vromoner al sud di Leskovik;

- Kosovo, le sorgenti di Pejë, Istog, Kllokot, Mitrovice ecc (Laçi 2006);

- I territori albanesi in Macedonia offrono le sorgenti di Diber (Banjisht e Kosovrast), quelle di Kumanova ecc;

- La valle di Presheva (territorio albanese nel Sud della Serbia) offre le sorgenti di Bujanoc e Sijarina (Ejupi 2013).

L'importanza delle sorgenti termo-minerali si associa ai loro valori curativi. Questi valori derivano dai sali contenuti e dalle temperature elevate ( L'Accademia delle Scienze 1991). Le acque minerali emergono dalle profondità della terra e contengono soprattutto gas di zolfo $\left(\mathrm{H}_{2} \mathrm{~S}\right)$ in quantità variabile.

L'Albania nonostante le piccole dimensioni, ha una serie di importanti sorgenti di importanza turistica e geotermale. Le sorgenti ed i pozzi delle acque termali in Albania sono localizzate in tre aree geotermiche: l'area geotermica di Kruja, l'area geotermica di Ardenica e l'aerea geotermica di Peshkopia.

Tabella 1. Le risorse termali e minerali in Albania

\begin{tabular}{|c|l|c|c|c|c|}
\hline \multirow{2}{*}{$N \mathbf{*}$} & \multirow{2}{*}{ Nome del sorgente e della regione } & & \multicolumn{2}{|c|}{ Le coordinate geografiche } & \\
\cline { 3 - 5 } & & Temp. & Largezza V & Lunghezza L & Afflusso Ils \\
\hline 1 & Mamuras 1 dhe 2 & $21-22$ & $41^{\circ} 35^{\prime} 24$ & $19^{\circ} 42^{\prime} 48^{\prime \prime}$ & 11.7 \\
\hline 2 & Shupal & 29.5 & $41^{\circ} 26^{\prime \prime} 9^{\prime \prime}$ & $19^{\circ} 55^{\prime} 24^{\prime \prime}$ & $<10.0$ \\
\hline 3 & Le terme di Elbasan (Llixha) & 60 & $41^{\circ} 02^{\prime}$ & $20^{\circ} 04^{\prime} 20^{\prime \prime}$ & 15.0 \\
\hline 4 & Hydrat, Elbasan & 55 & $41^{\circ} 1^{\prime} 20^{\prime \prime}$ & $20^{\circ} 05^{\prime} 15^{\prime \prime}$ & 18.0 \\
\hline
\end{tabular}




\begin{tabular}{|c|l|c|c|c|c|}
\hline 5 & Peshkopi & 43.5 & $41^{\circ} 42^{\prime} 10^{\prime \prime}$ & $20^{\circ} 27^{\prime} 15^{\prime \prime}$ & 14.0 \\
\hline 6 & II ponte di Katiu Langaricë, Përmet & 30 & $40^{\circ} 14^{\prime} 36^{\prime \prime}$ & $20^{\circ} 26^{\prime}$ & $>160.0$ \\
\hline 7 & Vromoneri, Sarandoporo, Leskovik & 26.7 & $40^{\circ} 5^{\prime} 54^{\prime \prime}$ & $20^{\circ} 40^{\prime} 18^{\prime \prime}$ & $>10.0$ \\
\hline 8 & Finiq, Saranda & 34 & $39^{\circ} 52^{\prime} 54^{\prime \prime}$ & $20^{\circ} 03^{\prime}$ & $<10.0$ \\
\hline 9 & Il ruscello di Holta, Gramsh & 24 & $40^{\circ} 55^{\prime} 30^{\prime \prime}$ & $20^{\circ} 09^{\prime} 24^{\prime \prime}$ & $>10.0$ \\
\hline 10 & Postenan, Leskovik & Sorgente di vapore & $40^{\circ} 10^{\prime} 24^{\prime \prime}$ & $20^{\circ} 33^{\prime} 36^{\prime \prime}$ & \\
\hline 11 & Kapaj, Mallakastër & $16.9-17.9$ & $40^{\circ} 32^{\prime} 30^{\prime \prime}$ & $19^{\circ} 48^{\prime} 42^{\prime \prime}$ & 12.0 \\
\hline 12 & Selenicë, Vlorë & 35.3 & $40^{\circ} 32^{\prime} 18^{\prime \prime}$ & $19^{\circ} 39^{\prime} 30^{\prime \prime}$ & $<10.0$ \\
\hline
\end{tabular}

Fonte: Atlante Geotermale dell'Albania (A.Frashëri,V. Cermak et al, 2004).

Nella seguente mappa si mostrano le sorgenti termo-minerali in Albania secondo la temperatura.

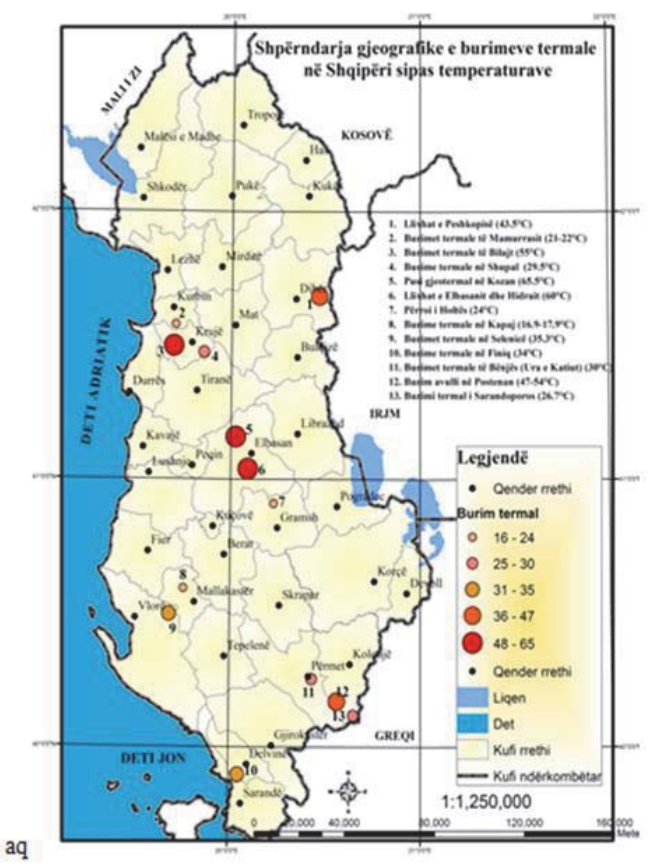

Figure 1. La distribuzione geografica delle sorgenti termali secondo le temperature in Albania

Come l'Albania anche il Kosovo ha risorse naturali che permettono lo sviluppo del turismo termale curativo. La composizione tanto complessa dei tipi delle rocce ha determinato numerose ricchezze minerarie (Laçi 2006). In Kosovo ci sono circa 30 sorgenti termali e minerali (Ministero dell' Ambiente e della Pianificazione Ambientale 2010) che vengono utilizzati per bere e per lavarsi.

In generale, le risorse idriche termo-minerali del Kosovo hanno temperature dal $17^{\circ} \mathrm{C}$ fino a $54^{\circ} \mathrm{C}$ (Bërxholi 2013) e la scala della mineralizzazione varia da $2-5 \mathrm{~g} / \mathrm{l}$. Si distinguono le sorgenti delle Terme di Peja, di Kllokoti, di Skënderaj ecc. Particolare interesse presentano le Terme di Kllokot. Le acque termali emergono nella valle di Binçe, sulla strada Ferizaj - Gjilan con temperature che variano tra $32^{\circ}-36^{\circ} \mathrm{C}$. Le acque termo-minerali di Kllokot si classificano di un' elevata importanza per la loro qualità di argilla ( pelloida). Non possiamo lasciare senza nominare le acque termali di Uglar con temperature fino a $29^{\circ} \mathrm{C}$ e quelle di Kmetovic con temperature $27^{\circ} \mathrm{C}$.

Sorgenti termali interesanti sono anche quelle di Peja all'est delle Bjeshke të Nemuna. Le sorgenti si trovano ai piedi delle montagne di Korenica in un'aerea tra Peja e Istog. Le acque termali hanno delle temperature che variano da $46^{\circ}-48^{\circ} \mathrm{C}$. Le Terme di Peja attirano visitatori per la cura di malattie come reumatismo, malattie delle ossa, della pelle ecc (Laçi 2006, L’Ufficio per la riabitilazione e la cura fisica Pejë 2012). 
Nei territori albanesi oltre confine TTSHPK ${ }^{2}$ mostrano interesse anche le acque termo- minerali della Valle di Presheva. Le sorgenti termo-minerali più importanti sono: Bujanoc, Banjka e le Terme di Sijarin. Le prime sono sorgenti ipertermali con temperature da $24,5^{\circ} \mathrm{C}$ fino a $43^{\circ} \mathrm{C}$ e pH 6,8 . Le acque termali e minerali di Sijarin riportano segni di attività vulcanica (Ejupi 2013) recente, con temperature fino a $75^{\circ} \mathrm{C}$ e un flusso di 8 Ilsec. Nella zona delle Terme di Sijarin ci sono 18 sorgenti termo-minerali. Vale la pena nominare anche le sorgenti termo-minerali nei territori della Macedonia dell'ovest come quelle di Kosovrast e Banjisht.

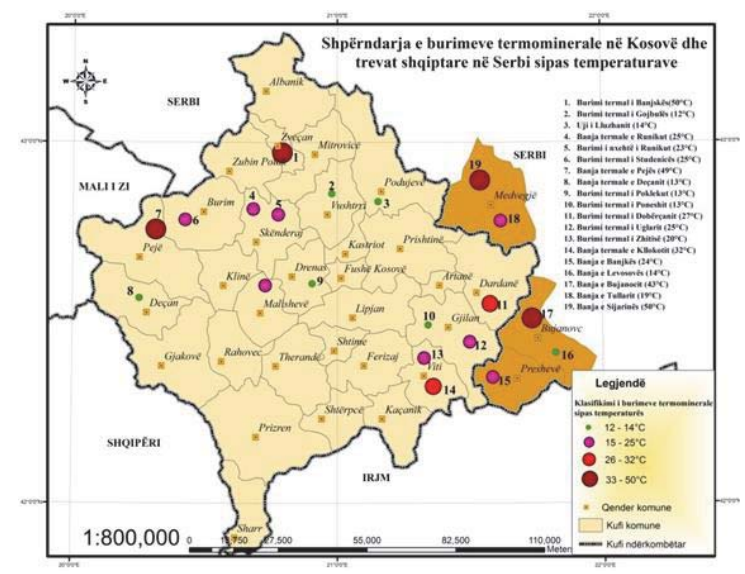

Figure 2. La distribuzione delle sorgenti termali secondo le temperature in Kosovo e nei territori albanesi della Serbia

\subsection{Le risorse umane}

Lo sviluppo del turismo viene sostenuto anche dalle risorse umane. In una data zona l'offerta turistica si presenta completa se si alternano le risorse naturali con quelle umane. Nel turismo curativo termale un'elevata importanza hanno non solo lerisorse minerali ma anche il patrimonio culturale, materiale e spirituale delle zone curative. Questi elementi favoriscono la permanenza piu' lunga dei visitatori nei centri curativi. Nello sviluppo del turismo curativo delle terme una notevole importanza offre anche la cultura sociale ed economica (potenzialitá umana) della zona e della comunitá che ospita i visitatori. Hanno importanza:

- l'infrastruttura, le strutture alberghiere;

- gli staff specializzati al servizio del cliente;

— il livello dell'istruzione in funzione dello sviluppo del turismo termale;

- l'influenza degli altri aspetti dell'economia in servizio del turismo delle terme;

- il livello degli interventi locali per assicurare lo sviluppo economico sostenibile delle zone termali ecc.

Il soggiorno dei turisti diventa più gradevole nelle zone con una ricca tradizione spirituale. Citiamo qui: I'ospitalità della comunità, la culinaria, i canti e le danze, senza dimenticare gli elementi del patrimonio materiale come: i costumi tradizionali, i prodotti di artigianato, gli strumenti musicali ecc.

Le potenzialita' culturali che favoriscono lo sviluppo del turismo curativo sono le rocche e i luoghi del culto religioso (le chiese, le moschee, il tekke). Non mancano i siti archeologici, le costruzioni etnografiche, il folclore, i costumi e le canzoni. Questi elementi sono parte importante dell'offerta turistica che i luoghi curativi offrono insieme all'offerta naturale.

L'offerta culturale in alcune zone é piu'attrattiva di quella naturale (curativa) favorita anche dalla continuitá durante tutto l'anno. II turismo curativo si intreccia anche con le visite nei luoghi di culto, i siti archeologici e quelli culturali) come a Elbasan, Peshkopi, Bënjë, Peja, Kumanova ecc. Vale nominare anche l'agriturismo a Bënjë, Peshkopi e Kllokot 
favorendo il fenomeno dell' escursionismo. Il fenomeno dell'escursionismo nel nostro territorio si concepisce con l'attivitá motoria dei visitatori verso i luoghi storici, culturali e archeologici inoltrate alle visite nei centri curativi termali vicini ad essi. I tour operator devono promuovere le visite dei turisti nelle città le quali nelle vicinanze hanno centri curativi.

\subsubsection{La cultura materiale in funzione dello sfruttamento dei valori curativi delle terme}

Il soggiorno dei visitatori nelle terme spesso si accompagna con delle visite agli oggetti più conosciuti della cultura materiale. Per i territori albanesi l'intreccio dei valori curativi delle terme con quelli culturali appare come segue:

Elbasan: I turisti visitano Elbasan il suo castello (Kala), come una delle fortezze costruite in pianura, l'unica nei Balcani, altre strutture religiose di culto, la tradizione e la cultura (Bevapi K., et al, 2003). Dopo le visite possono frequentare la zona curativa delleTerme di Tregan e di Hidraj. Lo spostamento può aver luogo nella direzione opposta. Dopo aver completato i trattamenti nei centri curativi, i visitatori possono trascorrere il loro tempo libero sempre a conoscenza di valori culturali e storici della zona di Elbasan. Elbasan offre ai visitatori una lunga lista di monumenti culturali e storici: Rrapi i Bezistanit (II Matusalemme di Bezistani), i ponti antichi, il castello di Mengel, la torre dell'orologio, la rocca di Sulova, la moschea reale ecc. Non possiamo non citare anche la moschea di Naziresha del XVII secolo, il hammam del XVI secolo, la chiesa di Santa Maria che é situata nel quartiere Kala, il museo etnografico, il grande tekke e il tekke di Baba Xhemal. Solo 4 chilometri dalla cittá dista il monastero di San Giovanni Vladimir. Recentemente un particolare interesse dimostra anche la scoperta archeologica al centro della cittá vicino al Matusalemme di Bezistan (Rrapi i Bezistanit) La scoperta rappresenta una basilica paleocristiana ( del V-VI a.c) ${ }^{3}$ e uno dei siti archeologici più interessanti.

Durante i lavori per rivitalizzare la torre sud-est della Kala di Elbasan sono stati scoperti tre stelle funebri illiriche (lapidi ). Si pensa che furono usate come materiale da costruzione. Queste stelle risalgono al. I- II secolo d. C.

La città di Elbasan ed i suoi dintorni offrono la migliore combinazione delle potenzialità culturali con quelle naturali in funzione dello sviluppo del turismo. Vicino alle Terme di Elbasan si trova Gjinar (Shpati), (Opportunity Albania 2011) zona con delle potenzialitá naturali e culturali. Le visite al centro curativo possono alternarsi con le visite in questa zona di attrazione turistica che si distingue per l'aria pulita e il clima salubre. La zona di Shpat offre anche importanti valori culturali come: la chiesa di Shelcan e di Valësh con le famose icone di Onufer e di Kostandin Shpataraku.

Oltre le terme di Tregan, Elbasan è noto anche per le acque termo-minerali del pozzo geotermico di Kozan. Queste acque, a differenza delle prime sono meno utilizzate per i bagni curativi, a prescindere che le prospettive per il futuro siano buone. II pozzo geotermico di Kozan é situato molto vicino alla città e al monastero di San Giovanni Vladimir a Shijon.

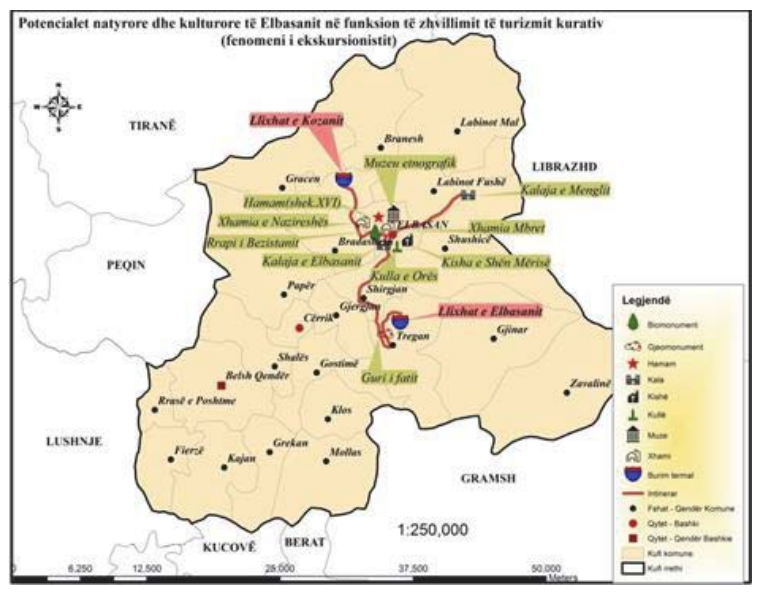

Figure 3. Le ricchezze naturali e culturali di Elbasan in funzione dello sviluppo curativo. 
Peshkopia: I più antichi valori storici della cittá si associano alla zona di Tekke. Essa rappresenta la parte storica della città, con uno stile specifico nelle costruzioni e nell'architettura. Una parte dei visitatori soggiorna nella cittá di Peshkopi ed ogni giorno si muove verso il centro curativo per concludere il ciclo della terapia curativa. Peshkopi offre visite nei musei storici della città e in altre strutture culturali: fortezze e luoghi religiosi di culto (moschee, chiese e tekke ). Molto ricco è anche il folclore, l'artigianato e l'etnografia della zona.

Fushë-Krujë (Bilaj): Dopo le terapie nei centri curativi i visitatori possono passare il tempo libero conoscendo le potenzialitá storiche e culturali di Kruja. Kruja é molto ricca in questo aspetto. Possiamo citare la fortezza di Kruja e vicino ad essa il caratteristico bazar, il Museo nazionale storico, il museo etnografico, l'orologio della torre, la chiesa vicino all'orologio della torre, il tekke di Dollmë, i hammam ecc. Tanti visitatori attira anche il museo dell'eroe nazionale Skanderbeg.

Përmet: I valori culturali e storici in questa zona sono tanti. Dimostrano interesse le strutture religiose di culto rappresentate dalle chiese di Leuse, di Kasina e di Bënjë. Le visite in queste chiese si alternano alle visite nelle antiche strutture archittetoniche come II Ponte di Kati e concludendosi nelle acque termo-minerali Bënjë. L'alternarsi delle potenzialitá culturali con quelle naturali sono le principali attrazioni in questa zona. I turisti anche quelli arrivati oltre confine visitano le acque termali di Bënjë, canyoni di Lengarica, l'acqua fredda di Këlcyra, "la pietra rocciosa" della cittá e apprezzano anche la culinaria della zona.

Leskovik: Leskovik é noto per il suo ricco patrimonio culturale. Tra le strutture più popolari e più visitate é la chiesa di Santa Maria Lashova dichiarato monumento culturale. E 'stata costruita nel 1800-1820 ed è famosa per le icone e le opere in legno delle figure della chiesa (Comune di Leskovik 2007). Oltre ad essa si distinguono la chiesa di Santa Maria nel villaggio Cërckë e la chiesa di San Sotir nell' altopiano di Melesin. Non mancano i siti religiosi di culto dell' ordine islamico Bektashi al centro della città. La prova della civiltà precoce sono le scoperte di insediamento illirico vicino alla città di Leskovik.

Kllokot: La zona di Amanarova dove si concentrano le sorgenti termo-minerali di Kllokot e di Uglar, è ricca di elementi del patrimonio materiale e spirituale. Le visite al centro curativo possono essere alternate con le visite a strutture culturali: il castello di Progragje, la chiesa di Santa Varvara, il monastero di San Nicola, le chiese, le icone, i tumuli, gli oggetti etnografici, i santuari, i tekke ecc (Kadriu 2012).

Peja: Peja è ricca di storia. Molti sono gli scavi archeologici, i momunenti cristiani (12) , i monumenti musulmani (23), i santuari e le torri dei bektashi. Nel centro della città si trova la vecchia moschea costruita nel 1471. Le visite turistiche a queste strutture si possono concludere con le visite nel centro curativo solo $12 \mathrm{~km}$ dalla città di Pejë. Le offerte culturali si alternano con quelle naturali. II soggiorno diventa attraente grazie alle passeggiate nella gola di Rugova.

Malishevë: Le terme di Malisheva sono conosciute in tutto il Kosovo. Nonostante le conseguenze delle guerre e dei problemi avuti negli anni passati, il villaggio Banjë riporta un ricco patrimonio culturale. Interesse presenta il mulino dei Begaj di 300 anni di storia. Nel comune di Malisheva ci sono ancora molte attrazioni naturali di interesse culturale per i visitatori come la cascata di Mirusha.

Dibër (Kosovrast e Banjishtë ): I visitatori che soggiornano in questi centri curativi possono fare delle visite a Dibër conoscendo le sue tradizioni culturali. II centro curativo offre ai visitatori gli elementi del patrimonio del passato attraverso l'antica piscina (1720) e le tracce della Via Egnatia . II centro ha adottato misure per regolamentare la piscina (struttura di valore storico e culturale) per attrarre visitatori e per migliorare l' immagine del centro.

Kumanovë: Kumanovo offre un ricco patrimonio materiale e spirituale. Le visite nelle terme di Kumanovo si alternano alle visite nelle strutture culturali, storiche e religiosi. L'antica Moschea di Eski nel centro storico, costruita nel 1751 (La guida di Kumanova), è la struttura più importante storico-culturale. Altri edifici importanti sono la chiesa di San Nicola, il Museo Nazionale, il Teatro Nazionale e molti siti archeologici nei suoi dintorni .

La Valle di Presheva: II patrimonio naturale viene rappresentato da questi oggetti: la grotta di lincës, la caverna dell'Orso, il Tronco di Qarr (Ejupi 2013) ecc. Un' importante offerta turistica e anche il patrimonio culturale rappresentato da siti archeologici, oggetti di culto (chiese, moschee, tekke e santuari), edifici residenziali (case a torre e stanze antiche (ode) , strutture pubbliche (locande, sale da tè, mulini e fontanelle) (Salihu 2013). Le visite possono concentrarsi anche ad importanti strutture culturali: la roccaforte di Presheva, la località di Përzhar, la fortezza di Bushtran e le tracce delle antiche fortezze dei Dardani.

II movimento turistico nei luoghi curativi ha come scopo l'apprezzamento dei motivi etno-sociali e culturali. Gli abiti tradizionali, i canti e le danze, gli strumenti musicali, le tradizioni e i costumi, le strutture religiose di culto, i proverbi e le leggende attirarano molti visitatori. 


\section{Le Conclusioni}

II turismo curativo termale é strettamente legato all'esistenza delle sorgenti termali e minerali. II territorio delle nostre terre con una variabile costruzione geologica offre molte fonti termo- minerali. II turismo curativo oltre le potenzialita' naturali viene sostenuto anche da quello culturale. I centri curativi hanno la possibilita' di offrire ai visistatori visite guidate nei luoghi del patrimonio culturale. Questo permette il prolungamento della loro permanenza in questi centri e favorisce lo sviluppo durante tutto l'anno.

Nei territori albanesi, lo sviluppo del turismo curativo è passato in diverse fasi ed è stato influenzato da sviluppi storici e politici. II potenziale turistico offerto, consente il suo sviluppo attuale. In un'epoca in cui il turismo è considerato come uno dei rami con più prospettiva nella nostra regione, diventa importante il ruolo che il turismo curativo svolge in aree con potenziale turistico curativo. Secondo l'importanza che ha, è necessario intensificare gli sforzi per rafforzare:

- Le politiche e strategie per lo sviluppo del turismo curativo;

- Lo sviluppo delle infrastrutture curative secondo gli standard europei;

- La promozione dei valori curativi lasciati in limbo;

- La preparazione di guide turistiche;

- Il coinvolgimento della comunità nello sviluppo del turismo;

- La qualifica professionale della nuova generazione con nuovi concetti sul turismo curativo e di benessere;

- Gli investimenti locali e stranieri nel settore del turismo curativo ecc.

\section{References}

Frashëri A.,Cermak V. et al,(2004). Atlante Geotermale dell'Albania, Universitá Politecnico di Tirana, Facoltá di Geologia e delle Miniere, 18.

Bagnoti, (2009). Geografia del turismo, Dal turismo di elite al turismo di massa, cap I, 2.

Becheri E.,et al,(2006). Le terme tra sostenibilita è compatibilitá, 7.

Bërxholi A., (2013). La geografia dei territori etnici albanesi, 221.

Bevapi K., et al, (2003). Elbasani Enciclopedia, II comune della cittá, 149. http://www.elbasani.gov.al/

Doka Dh., Draçi B., (2009). La geografia del turismo, Casa editrice del libro universitario, 55-75.

Ejupi A., (2013). La valle di Presheva-studio geografico regionale, 52.

Il comune di Tregan, 2012

Il comune di Petran, 2012

II comune di Peshkopi 2012

L'Accademia delle Scienze (1991), Geografia fisica dell'Albania, 258-259.

Laçi S., (2006). La geografia del Kosovo e dei territori albanesi oltre le confini della Repubblica dell'Albania, 59-183.

L'Ufficio per la riabitilazione e la cura fisica, Ahmet Blakaj, Peja maggio 2012.

L'Istituto dei monumenti in Albania.

Leskovik (2007). Piano d' azione nell' ambiente, comune di Leskovik, 23.

La guida di Kumanova, 7.

Kadriu Sh.,(2012). Gjilan, 360-362.

Muhaxheri N., (1989). La politica del prodotto turistico come base per lo sviluppo del turismo nella regione di Peja, "Ramiz Sadiku Prizren" Pejë, 81.

Ministero dell' Ambiente e della Pianificazione Ambientale 2010, risorse idriche del, 5.

Opportunity Albania (2011), Benvenuti a Shpat, guida turistica, Elbasan.

Piano di sviluppo del turismo, comune di Tregan, Petran, FSHZH, II centro di ricerca e di sviluppo, giugno 2009, 7.

Salihu Xh., (2013). II patrimonio culturale e naturale a Presheva, (http://presheva.com/)

(http://www.dec.unich.it 